\title{
Critical probability bounds for two-dimensional site percolation models
}

\author{
Tomasz Luczak $† \ddagger$ and John C Wierman $\uparrow \S \|$ \\ $\dagger$ Institute for Mathematics and its Applications, University of Minnesota, Minneapolis, \\ MN 55455, USA \\ $\ddagger$ Adam Mickiewicz University, Poznań, Poland \\ $\S$ Mathematical Sciences Department, Johns Hopkins University, Baltimore MD 21218, \\ USA
}

Received 12 February 1988

\begin{abstract}
We present three techniques for determining rigorous bounds for site percolation critical probabilities of two-dimensional lattices. A technique for finding lower bounds for critical probabilities of bipartite graphs is used to show that $p_{\mathrm{c}}(D) \geqslant 0.5020$ for the dice lattice $D$. Combining this method with Kesten's duality result simplifies Toth's derivation of the lower bound $p_{c}(S) \geqslant 0.5034$ for the square lattice $S$. We also present a technique for deriving upper bounds for bipartite graphs. A technique of grouping sites is used to derive upper bounds for the critical probability of the hexagonal lattice $H: p_{c}(H) \leqslant 0.8079$ and $p_{c}(H) \leqslant \sqrt{p_{c}(S)}$. The grouping technique is applied to the dice lattice to find the upper bound $p_{c}(D) \leqslant 0.7937$.
\end{abstract}

\section{Introduction}

For many years following the introduction of percolation models by Broadbent and Hammersley (1957) the major focus of mathematical percolation theory was the exact determination of critical probabilities (also called percolation thresholds). A heuristic method of Sykes and Essam (1964) conjectured the value $\frac{1}{2}$ for the square lattice bond model and the triangular lattice site model, $2 \sin (\pi / 18) \approx 0.347$ for the triangular lattice bond model, and $1-2 \sin (\pi / 18)$ for the hexagonal lattice bond model. Rigorous proofs were obtained much later by Kesten $(1980,1982)$ and Wierman (1981). Wierman (1984) derived exact bond percolation critical probabilities for an additional dual pair of lattices. Even though very little is known about the relationship of the critical probability to the detailed structure of the lattice, much recent work on percolation has emphasised the critical exponents of the percolation model, which are believed to be identical for large classes of lattices (called universality classes). Further study of the critical probability is necessary to obtain a complete understanding of percolative behaviour.

Several techniques have been used to gain information about site percolation critical probabilities, as follows.

Connectivity constant. For a fixed vertex $v \in G$, let $f_{n}$ denote the number of self-avoiding paths in $G$ of length $n$ starting from $v$. The connectivity constant, denoted $\lambda(G)$, is

|| Research supported in part by the National Science Foundation grant DMS-8403646.

$0305-4470 / 88 / 143131+08 \$ 02.50$ (C) 1988 IOP Publishing Ltd 
defined by

$$
\lambda(G)=\liminf _{n \rightarrow \infty} f_{n}^{1 / n} .
$$

(For a connected graph $G, \lambda(G)$ is independent of the initial vertex $v$.) Hammersley (1957) showed that $p_{c}(G) \geqslant 1 / \lambda(G)$. A crude lower bound is provided by $p_{\mathrm{c}}(G) \geqslant$ $1 /(\Delta(G)-1)$ where $\Delta(G)$ is the maximum degree of a vertex in $G$, since $\lambda(G) \leqslant$ $\Delta(G)-1$.

Bond-site transformation. By the bond-to-site transformation (Fisher 1961), a bond percolation model on a graph $G$ is converted into an equivalent site percolation model on the line graph (or covering graph) of $G$, so the two models have equal critical probabilities. For example, the site percolation critical probability of the Kagome lattice is equal to the bond percolation critical probability of the hexagonal lattice, which is known to be exactly $1-2 \sin (\pi / 18) \approx 0.6527$. However, the bond-to-site transformation is not invertible, so there exist site models for which there are no corresponding bond models. Thus, rigorous solutions for all bond models would still not supply solutions for all site models.

Bond model bounds. Hammersley (1961) showed that for any graph, the bond percolation critical probability is a lower bound for the site percolation critical probability. For an alternative proof, see Oxley and Welsh (1979). Exact bond percolation critical probabilities of several lattices (mentioned above) are exactly known, and, except for the square and triangular lattices, provide the best known rigorous lower bound for the site percolation critical probability.

Inclusion principle. If $G_{1} \subseteq G_{2}$, the critical probability of $G_{1}$ is at least as large as that of $G_{2}$. This 'inclusion principle' provides critical probability relationships by comparing lattices. Kesten (1982) has shown that, under certain regularity assumptions, the inequality is strict, and Menshikov (1987) has developed a method for finding a lower bound on the difference. Using this method, Zuev (1987) has found that $0.5095 \leqslant$ $p_{c}(S) \leqslant 0.6819$ for the square lattice.

Duality. Sykes and Essam (1964) introduced the concept of matching pairs of graphs to extend duality theory to site percolation models. Kesten (1982) proved that site percolation models of matching graphs sum to one if the graphs are periodic and have at least one axis of symmetry. Obviously, a bound on the critical probability of one graph implies a bound on the critical probability of the matching graph (in the opposite direction).

In $\S 2$ of this paper, we introduce three additional techniques for obtaining bounds for critical probabilities. For bipartite lattices, we provide a method for constructing a related lattice, plus two techniques using this related lattice which yield lower and upper bounds for the critical probability. In some cases, results may be improved by using the fact that the critical probabilities of a matching pair of lattices sum to one. Toth (1985) uses a similar argument to find a lower bound of 0.5034 for the square lattice site percolation critical probability. Our argument gives a simpler proof for the same bound. A third technique, involving grouping of vertices, is used to obtain an upper bound of 0.8079 for the critical probability of the hexag inal lattice, and 0.7937 for the dice lattice. 
Section 3 summarises the results of applying these techniques to the square, hexagonal and dice lattices, which are used as examples in $\S 2$. We believe that our results provide the best upper bound for the hexagonal and dice lattices, and best lower bound for the dice lattice. To the best of our knowledge, the best known rigorous numerical bounds are

$$
\begin{aligned}
& 0.5020 \leqslant p_{c}(D) \leqslant 0.7937 \\
& 0.6527 \leqslant p_{c}(H) \leqslant 0.8079 \\
& 0.5095 \leqslant p_{c}(S) \leqslant 0.6819 .
\end{aligned}
$$

\section{Techniques and examples}

\subsection{Bipartite graphs}

A bipartite graph $G$ is a graph for which the vertex set can be partitioned into two sets, $A$ and $B$, in such a way that every edge of $G$ has one endpoint in $A$ and one endpoint in $B$. An equivalent characterisation is that $G$ is bipartite if and only if it contains only even cycles.

Consider a bipartite lattice $L$ with bipartition $(A, B)$. Define $L_{A}$ to be the lattice with vertex set $A$ with edges between a pair of vertices of $A$ which have at least one common neighbour in $L$. $L_{B}$ may be defined similarly.

Since existence of an infinite open path in $L$ implies existence of an infinite open path in $L_{A}$, then

$$
p_{\mathrm{c}}(L) \geqslant p_{\mathrm{c}}\left(L_{\mathrm{A}}\right) \text {. }
$$

A heuristic argument due to Shalitin (1982) suggests that if $L_{A}=L_{B}$ the inequality

$$
p_{c}(L) \leqslant \sqrt{p_{c}\left(L_{A}\right)}
$$

holds, and that $p_{\mathrm{c}}(L)$ is near this upper bound.

For a bipartite lattice $L$, we construct another related lattice as follows. Suppose that the degree of each vertex in $A$ is $a$, and the degree of each vertex in $B$ is $b$. Let $L^{+}$denote the lattice obtained by inserting a vertex on each edge of $L$. In the two methods described below, we essentially replace each vertex $v$ of $B$ by the set of vertices inserted on edges incident to $v$, so we will refer to the inserted vertices as parts of $v$.

\subsection{Lower bounds for bipartite graphs}

To obtain a lower bound for the critical probability of $L$, we relate site percolation configurations on $L, L^{+}$and $L_{A}$ by the following procedure.

(i) Generate a configuration on $L^{+}$by declaring each vertex in $A$ to be open with probability $p$, and each part of each vertex of $B$ to be open with probability $p^{1 / b}$. (Each vertex of $B$ is open with probability one.)

(ii) Construct a configuration on $L$ by declaring each vertex in $A$ to be open if it is open in $L^{+}$, and each vertex in $B$ to be open if all its parts are open in $L^{+}$.

(iii) Construct a configuration on $L_{A}$ by declaring each vertex in $A$ to be open if it is open in $L^{+}$and at least two of the neighbouring parts of vertices of $B$ are open. 
By construction, every vertex in $L$ is open with probability $p$, and every vertex in $L_{A}$ is open with probability

$$
p\left[\sum_{i=2}^{a}\left(\begin{array}{l}
a \\
i
\end{array}\right) p^{i / b}\left(1-p^{1 / b}\right)^{a-i}\right] .
$$

Suppose $p>p_{\mathrm{c}}(L)$. Then, with probability one, there exists an infinite self-avoiding open path $v_{0}, v_{1}, v_{2}, \ldots$ in $L$. In this case, either $v_{1}, v_{3}, v_{5}, \ldots$ or $v_{2}, v_{4}, v_{6}, \ldots$ is an infinite self-avoiding open path in $L_{A}$. Since each vertex of $L_{A}$ is open with probability

$$
p\left[\sum_{i=2}^{a}\left(\begin{array}{l}
a \\
i
\end{array}\right) p^{i / b}\left(1-p^{1 / b}\right)^{a-i}\right]
$$

we have

$$
p\left[\sum_{i=2}^{a}\left(\begin{array}{l}
a \\
i
\end{array}\right) p^{i / b}\left(1-p^{1 / b}\right)^{a-i}\right] \geqslant p_{\mathrm{c}}\left(L_{\mathrm{A}}\right) .
$$

Therefore, $p_{\mathrm{c}}(L) \geqslant p_{0}$, where $p_{0}$ is the unique root of the equation

$$
p\left[\sum_{i=2}^{a}\left(\begin{array}{l}
a \\
i
\end{array}\right) p^{i / b}\left(1-p^{1 / b}\right)^{a-i}\right]=p_{c}\left(L_{A}\right) .
$$

Note that $p$ is multiplied by a factor which is strictly smaller than one, so we obtain the strict inequality $p_{\mathrm{c}}(L)>p_{\mathrm{c}}\left(L_{A}\right)$.

We now illustrate with a few examples.

Hexagonal lattice. Let $H$ denote the hexagonal lattice. Then $H_{A}$ and $H_{B}$ are both the triangular lattice, so $a=b=3$ and $p_{c}\left(H_{A}\right)=\frac{1}{2}$ (see Kesten 1982). Solving the equation

$$
p\left[3 p^{2 / 3}\left(1-p^{1 / 3}\right)+p\right]=\frac{1}{2} .
$$

we obtain the lower bound

$$
p_{\mathrm{c}}(H) \geqslant 0.5477 \text {. }
$$

Dice lattice. Let $D$ denote the dice lattice. $D_{A}$ is the triangular lattice, as in the first example, but here $a=3$ and $b=6$. We obtain the lower bound $p_{c}(D) \geqslant 0.5009$ by solving the equation

$$
p\left[1-\left(1-p^{1 / 3}\right)^{6}-6 p^{1 / 3}\left(1-p^{1 / 3}\right)^{5}\right]=\frac{1}{2} .
$$

However, by a slight modification of the technique, the bound can be improved to $p_{c}(D) \geqslant 0.5020$, the root of the equation

$$
p\left[1-\left(1-p^{1 / 3}\right)^{6}-6 p^{1 / 3}\left(1-p^{1 / 3}\right)^{5}-2 p^{2 / 3}\left(1-p^{1 / 3}\right)^{4}\right]=\frac{1}{2}
$$

Square lattice. Let $S$ denote the square lattice. Then $S_{A}$ is the matching lattice of the square lattice, denoted $S^{M}$, and $a=b=4$. Since the critical probability of $S^{M}$ is unknown, we continue by using $p_{\mathrm{c}}(S)+p_{\mathrm{c}}\left(S^{M}\right)=1$, which is a consequence of Kesten's (1982) fundamental theorem. The previous analysis shows that for $p>p_{c}(S)$,

$$
p\left[6 p^{1 / 2}\left(1-p^{1 / 4}\right)^{2}+4 p^{3 / 4}\left(1-p^{1 / 4}\right)+p\right] \geqslant p_{c}\left(S^{M}\right)
$$

Thus, if $p>p_{\mathrm{c}}(S)$, then

$$
p+p\left[6 p^{1 / 2}\left(1-p^{1 / 4}\right)^{2}+4 p^{3 / 4}\left(1-p^{1 / 4}\right)+p\right] \geqslant p_{\mathrm{c}}(S)+p_{\mathrm{c}}\left(S^{M}\right)=1 .
$$


Finally, we obtain the lower bound $p_{c}(S) \geqslant 0.5034$, which is the root of the equation

$$
p+p\left[6 p^{1 / 2}\left(1-p^{1 / 4}\right)^{2}+4 p^{3 / 4}\left(1-p^{1 / 4}\right)+p\right]=1 \text {. }
$$

This bound was obtained by a somewhat more complicated procedure by Toth (1985).

\subsection{Upper bounds}

Let $0 \leqslant q \leqslant 1$. In $L^{+}$, let each of the parts of vertices in $B$ be open independently with probability $q$, and each vertex of $A$ be open independently with probability

$$
p(q)=\sum_{i=2}^{a}\left(\begin{array}{l}
a \\
i
\end{array}\right) q^{i}(1-q)^{a-i} .
$$

Define configurations on $L$ and $L_{A}$ as follows.

(i) Construct a configuration on $L$ by declaring each vertex in $A$ to be open if it is open in $L_{A}$, and each vertex in $B$ to be open if at least two of its parts are open.

(ii) Construct a configuration on $L_{A}$ by declaring each vertex to be open if it is open in $L^{+}$and all parts of its neighbours that lie on edges incident to it are open.

With these relationships, each vertex of $L$ is open with probability $p(q)$, and each vertex of $L_{A}$ is open with probability $q^{b} p(q)$.

If $q^{b} p(q) \geqslant p_{c}\left(L_{A}\right)$, then with probability one there exists an infinite open path $v_{0}, v_{1}, v_{2}, v_{3}, \ldots$ in $L_{A}$. This implies the existence of an infinite open path $v_{0}, w_{0}, v_{1}, w_{1}, v_{2}, w_{2}, \ldots$ in $L$, so we have $p(q) \geqslant p_{\mathrm{c}}(L)$. Thus,

$$
p_{\mathrm{c}}(L) \leqslant \sum_{i=2}^{a}\left(\begin{array}{l}
a \\
i
\end{array}\right) q_{0}^{i}\left(1-q_{0}\right)^{a-i}
$$

where $q_{0}$ is the root of the equation

$$
q^{b}\left[\sum_{i=2}^{a}\left(\begin{array}{l}
a \\
i
\end{array}\right) q^{i}(1-q)^{a-i}\right]=p_{\mathrm{c}}\left(L_{A}\right) \text {. }
$$

Example. For the hexagonal lattice, we obtain $q_{0}=0.8182$ as the root of

$$
q^{b}\left[q^{3}+3 q^{2}(1-q)\right]=\frac{1}{2}
$$

which implies the upper bound

$$
p_{\mathrm{c}}(H) \leqslant q_{0}^{3}+3 q_{0}^{2}\left(1-q_{0}\right)=0.9129 \text {. }
$$

\subsection{Grouping}

By grouping the sites of a lattice, then studying the connectivity between groups, one can often relate a graph with unknown critical probability to a graph for which the critical probability is either known or bounded appropriately. A rigorous bound is obtained from the relationship that is found. We illustrate with four examples.

Square lattice. Choose pairs of adjacent sites of $S$ as groups, as shown in figure 1, and consider a group to be open if both sites are open. Two groups are adjacent if there is an edge with one endpoint in each group, producing the triangular lattice $T$. If there is an infinite path of open groups in $T$, then there is an infinite open path in $S$. Thus, $p^{2}>p_{\mathrm{c}}(T)=\frac{1}{2}$ implies that $p>p_{\mathrm{c}}(S)$, so we obtain the upper bound $p_{\mathrm{c}}(S) \leqslant 1 / \sqrt{2} \approx$ 0.7072 . 


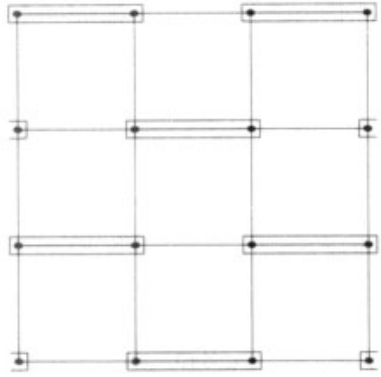

Figure 1. Square lattice.

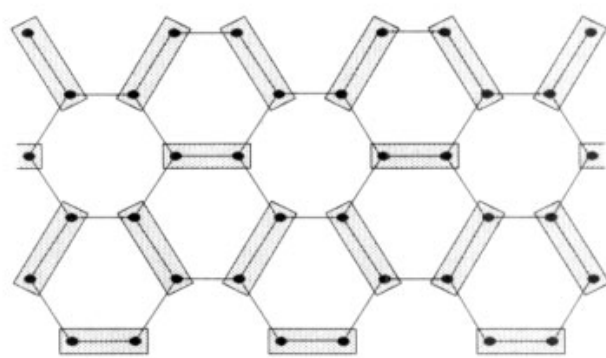

Figure 2. Hexagonal lattice.

Hexagonal lattice. First, consider groups of two sites as in figure 2, and let a group be open if both sites are open. Two groups are adjacent if there is an edge with one endpoint in each group, so the resulting lattice is the Kagome lattice $K$. Thus, $p^{2}>p_{\mathrm{c}}(K)=1-2 \sin (\pi / 18) \approx 0.6527$ implies that $p>p_{\mathrm{c}}(H)$, from which we obtain $p_{\mathrm{c}}(H) \leqslant 0.8079$.

If instead we group the sites as in figure 3 , we find that the groups are connected as in the square lattice $S$. Thus, $p^{2}>p_{\mathrm{c}}(S)$ implies that $p>p_{\mathrm{c}}(H)$, producing the

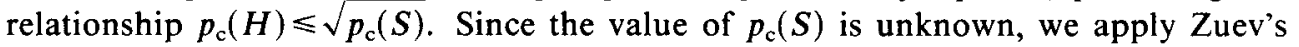
(1987) upper bound of 0.6819 to obtain $p_{\mathrm{c}}(H) \leqslant 0.8258$. Although this bound is larger than that found in the previous paragraph, it is improvable as better bounds are found for $p_{\mathrm{c}}(S)$. Using the estimated value of $p_{\mathrm{c}}(S) \approx 0.5927$ yields a value of $\sqrt{p_{\mathrm{c}}(S)} \approx 0.7699$.

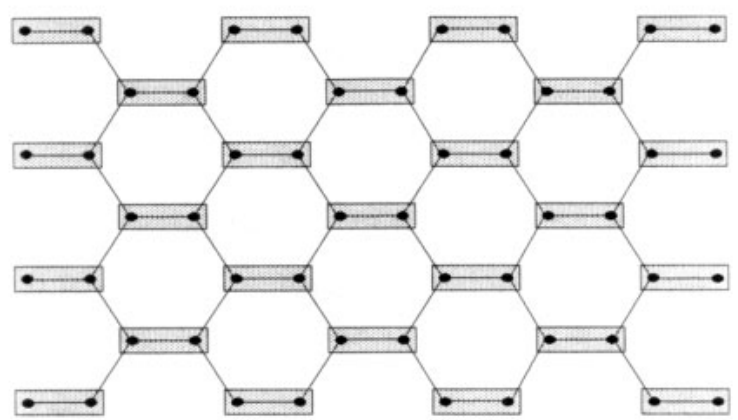

Figure 3. Hexagonal lattice with groups connected as in the square lattice $S$.

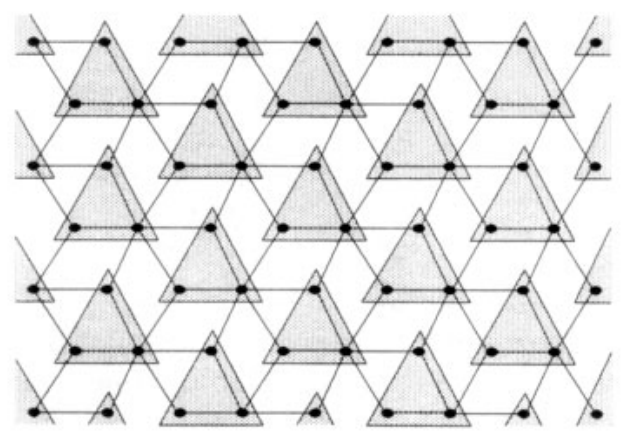

Figure 4. Dice lattice grouped as the triangular lattice $T$. 
Dice lattice. The dice lattice contains the hexagonal lattice, so by the inclusion principle and Kesten's (1982) strict inequality result, we have $p_{c}(D) \leqslant 0.8079$ and $p_{c}(D)<\sqrt{p_{c}(S)}$ from the hexagonal lattice bounds given above. We obtain another upper bound by considering groups of three sites as in figure 4. Each group is open if all three of its sites are open, and the resulting lattice is the triangular lattice $T$. An infinite path of open groups implies an infinite path of open sites in $D$. Thus, $p^{3}>p_{\mathrm{c}}(T)=\frac{1}{2}$ implies $p>p_{c}(D)$, from which we obtain the bound $p_{c}(D) \leqslant\left(\frac{1}{2}\right)^{1 / 3}=0.7937$.

\section{Summary}

We first collect the results for the three lattices used as examples in $\S 2$.

Square lattice. Many researchers have estimated the critical probability of the square lattice site percolation model by Monte Carlo simulation and renormalisation methods. See Ziff and Sapoval (1986) for a compilation of estimates for $p_{\mathrm{c}}(S)$, with a consensus for a value near 0.5927 . We obtain a lower bound of 0.5034 by the bipartite graph method, a value obtained earlier by Toth (1985). The best bounds, due to Zuev (1987), are

$$
0.5095 \leqslant p_{\mathrm{c}}(S) \leqslant 0.6819 \text {. }
$$

Hexagonal lattice. The critical probability of the hexagonal lattice is estimated_as 0.6962 (see Stauffer (1985) p 17) while Shalitin's conjectured upper bound is $1 / \sqrt{2} \approx$ 0.7072 . In $\S 2$, we derived lower bounds by the bipartite graph method $(0.5477)$, and a better lower bound may be found by the grouping method (0.6044). However, the best lower bound is the critical probability of the bond percolation model on the hexagonal lattice, which is $1-2 \sin (\pi / 18) \approx 0.6527$. The bipartite graph method yields an upper bound of 0.9129 , while the grouping technique gives upper bounds of 0.8079 and $\sqrt{p_{c}(S)}$. Thus,

$$
0.6527 \leqslant p_{\mathrm{c}}(H) \leqslant \min \left\{0.8079, \sqrt{p_{\mathrm{c}}(S)}\right\} \text {. }
$$

Dice lattice. By the bipartite graph method, we obtained lower bounds of 0.5009 and 0.5020 . Upper bounds of 0.7937 and $\sqrt{p_{c}(S)}$ were obtained in $\S 2.4$ by the grouping technique. Hence,

$$
0.5020 \leqslant p_{\mathrm{c}}(D) \leqslant \min \left\{0.7937, \sqrt{p_{\mathrm{c}}(S)}\right\} .
$$

The most notable observation is the lack of sharpness of the bounds. Other than the isolated cases that were exactly solved due to special properties, the bounds do not even determine the first digit for any of the lattices.

Note also that both the bipartite graph lower bound technique and the grouping technique provide the best bound known for at least one of the lattices.

Despite many advances within the thirty years since the introduction of percolation models, the exact determination of critical probabilities and of accurate bounds for critical probabilities remains an interesting and challenging open problem.

\section{References}

Broadbent S R and Hammersley J M 1957 J. R. Stat. Soc. B 53 629-45

Fisher M 1961 J. Math. Phys. 2 620-7 
Hammersley J M 1957 Ann. Math. Stat. 28 790-5

1961 J. Math. Phys. 2 728-33

Kesten H 1980 Commun. Math. Phys. 74 41-59

1982 Percolation Theory for Mathematics (Basle: Birkhauser)

Menshikov M V 1987 Usp. Mat. Nauk 42 239-40

Oxley J G and Welsh D J A 1979 J. Appl. Prob. 16 526-40

Shalitin D 1982 J. Stat. Phys. 28 99-110

Stauffer D 1985 Introduction to Percolation Theory (London: Taylor and Francis)

Sykes M F and Essam J W 1964 J. Math. Phys. 5 1117-27

Toth B 1985 Z. Wahrsch. verw. Geb. 69 19-22

Wierman J C 1981 Adv. Appl. Prob. 13 293-313

1984 J. Phys. A: Math. Gen. 17 1525-30

Ziff R M and Sapoval B 1986 J. Phys. A: Math. Gen. 19 L1169-72

Zuev S A 1987 Theor. Prob. Appl. 32 606-9 